Reprod. Nutr. Dévelop., 1987, 27 (1 B), 293-294.

\title{
Particularité de l'antagonisme des acides aminés à chaîne ramifiée chez l'agneau préruminant
}

Isabelle PAPET, D. BREUILLE, M. ARNAL

Laboratoire d'Etude du Métabolisme Azoté,

I.N.R.A. et C.N.R.S. U.A. 0411 23, Theix 63122 Ceyrat, France.

Summary. Preruminant lambs were fed a milk substitute containing different levels of Lleucine : 2.3 (control), $5.2,10.6$ or $12.6 \mathrm{~g} / 100 \mathrm{~g} \mathrm{DM}$. Increasing dietary L-leucine to 10.6 and $12.6 \mathrm{~g} / 100 \mathrm{~g} \mathrm{DM}$ markedly decreased food intake. All excess L-leucine in the diet resulted in an increase in plasma free L-leucine. Surprisingly, this did not change the plasma free L-valine and L-isoleucine levels. Thus, excessive dietary L-leucine appeared to have a different effect in lambs than in rats, chicks, pigs and men.

Les interactions entre les acides aminés à chaîne ramifiée (AACR) ont fait l'objet de nombreux travaux chez les monogastriques (Harper et al., 1984). En revanche, les rares études effectuées chez les ruminants indiquent que le métabolisme des AACR est particulier chez le mouton. Par exemple, Lindsay et Buttery (1980) ont émis l'hypothèse que leur catabolisme musculaire serait plus faible que chez le rat. Le but de cette expérience est de déterminer l'effet de différents excès alimentaires de L-leucine sur l'ingestion et les teneurs plasmatiques en AACR chez l'agneau préruminant.

Matériel et méthodes. Dix-sept agneaux mâles de race Limousine reçoivent un lait de remplacement dont la matière sèche (MS $=16 \%$ ) contient $24 \%$ de protéines $(N \times 6,25)$ apportées exclusivement par de la poudre de lait écrémé et $2,3 \%$ de L-leucine. Celui-ci est distribué vers $9 \mathrm{~h} 30$ et $16 \mathrm{~h} 00$. Les consommations et croissances moyennes des animaux sont respectivement de $270 \mathrm{~g} \mathrm{MS} / \mathrm{j}$ et $280 \mathrm{~g} / \mathrm{j}$ entre les âges de 1 et 2-3 semaines. Les agneaux, âgés de 2-3 semaines, sont séparés en 3 lots $A$, B et $C$ de respectivement 6,5 et 6 agneaux et reçoivent pendant 2 jours $(J+1$ et $J+2)$ le lait supplémenté en L-leucine contenant alors respectivement 5,$2 ; 10,6 ; 12,6 \mathrm{~g}$ de L-leucine $/ 100 \mathrm{~g} \mathrm{MS}$. Les quantités ingérées sont mesurées pendant 6 jours $(\mathrm{J}-3$ à $\mathrm{J}+2)$ dont 4 avant l'excès de L-leucine. Des prélèvements sanguins (veine jugulaire) sont effectués les jours $\mathrm{J} 0$ à $\mathrm{J}+2$, $4,5 \mathrm{~h}$ après le début du repas matinal au cours duquel les agneaux ingèrent en 0,5 à 1 h, 20 à $30 \%$ de leur consommation journalière. Les animaux sont privés de nourriture entre la fin du repas et la prise de sang. Les acides aminés libres plasmatiques sont dosés sur un analyseur automatique.

TABL. 1. - Consommation journalière des agneaux en fonction de l'excès alimentaire en L-leucine.

\begin{tabular}{cccc}
\hline Lot & $\mathrm{J} 0$ & $\mathrm{~J}+1$ & $\mathrm{~J}+2$ \\
\hline $\mathrm{A}$ & $98 \pm 12^{*}$ & $94 \pm 9$ & $98 \pm 18$ \\
\hline $\mathrm{B}$ & $92 \pm 16$ & $92 \pm 13$ & $86 \pm 9^{* *}$ \\
\hline $\mathrm{C}$ & $97 \pm 11$ & $76 \pm 9^{* *}$ & $67 \pm 29^{* *}$ \\
\hline
\end{tabular}

* : moyennes \pm écarts-types. Les moyennes sont exprimées en pourcentage des valeurs moyennes mesurées les trois jours $J-3$ à $J-1 ;{ }^{* *}$ : valeurs significativement différentes de $100 \%$ (test $t$ pour données appariées, $P<0,05)$. 
Résultats et discussion. L'augmentation de la teneur alimentaire en L-leucine de 2,3 à $5,2 \mathrm{~g} / 100 \mathrm{~g} \mathrm{MS}$ (lot A) n'induit pas de modification significative de la consommation. En revanche, l'ingestion est significativement réduite de $13 \%$ pour le lot $B\left(10,6 \mathrm{~g}\right.$ de L-leucine/100 $\mathrm{g}$ MS) le $2^{\mathrm{e}}$ jour d'excès et de 24 et $30 \%$ pour le lot $C\left(12,6 \mathrm{~g}\right.$ de L-leucine/100 $\mathrm{g}$ MS) respectivement le $1^{2}$ er et $2^{\circ}$ jour d'excès (tabl. 1). La teneur plasmatique en L-leucine libre augmente avec l'importance de l'excès de L-leucine dans l'aliment (tabl. 2). Toutefois, les teneurs plasmatiques en L-valine et L-isoleucine libres ne varient pas en fonction de l'apport alimentaire de L-leucine.

TABL. 2. - Teneurs des acides aminés à chaîne ramifiée libres plasmatiques en fonction de la teneur alimentaire de L-leucine.

\begin{tabular}{ccccc}
\hline Lot & Jour & $\begin{array}{c}\text { L-leucine } \\
\text { (mg/100 ml plasma) }\end{array}$ & $\begin{array}{c}\text { L-isoleucine } \\
(\mathrm{mg} / 100 \mathrm{ml} \text { plasma })\end{array}$ & $\begin{array}{c}\text { L-valine } \\
(\mathrm{mg} / 100 \mathrm{ml} \text { plasma })\end{array}$ \\
\hline A, B, C & $\mathrm{J} \mathrm{O}$ & $2,1 \pm 0,5^{*}$ & $1,1 \pm 0,2$ & $3,0 \pm 0,6$ \\
\hline A & $\mathrm{J}+1$ et $\mathrm{J}+2$ & $5,7 \pm 1,0$ & $1,1 \pm 0,2$ & $2,5 \pm 0,5$ \\
\hline B & $\mathrm{J}+1$ et $\mathrm{J}+2$ & $6,0 \pm 1,0$ & $1,0 \pm 0,2$ & $2,5 \pm 0,4$ \\
\hline C & $\mathrm{J}+1$ et $\mathrm{J}+2$ & $9,9 \pm 3,3$ & $0,9 \pm 0,1$ & $2,7 \pm 0,8$ \\
\hline
\end{tabular}

* moyennes \pm écarts-types.

La réduction de l'ingestion induite par les excès alimentaires importants de L-leucine a été décrite chez plusieurs espèces (rat : Harper et al., 1970 ; poulet : Calvert et al., 1982) excepté chez le chat (Hargrove et al., 1984). Ce phénomène est caractéristique de l'antagonisme nutritionnel entre les AACR. Chez toutes les espèces étudiées, mis à part le chat, l'augmentation de la teneur plasmatique en L-leucine observée avec les régimes contenant un excès de L-leucine s'accompagne d'une diminution des teneurs plasmatiques en L-valine et $L$-isoleucine libres (Harper et al., 1984). Cette chute des teneurs plasmatiques en L-valine et Lisoleucine est due à la stimulation du catabolisme des AACR par la L-leucine (enzymes de dégradation communs aux trois AACR). L'absence de diminution des teneurs plasmatiques en L-valine et L-isoleucine chez l'agneau soumis à un excès alimentaire en L-leucine indique indirectement que le catabolisme des AACR et/ou sa régulation seraient particuliers chez l'agneau préruminant.

Les excès alimentaires en L-leucine réalisés chez l'agneau préruminant révèlent un antagonisme particulier entre les AACR. Cette particularité ne serait donc pas liée à l'état ruminant comme le soulignaient Lindsay et Buttery (1980) mais plutôt à l'espèce animale.

Calvert C. C., Klasing K. C., Austic R. E., 1982. J. Nutr., 112, 627-635.

Hargrove D. M., Rogers Q. R., Morris J. G., 1984. Fed. Proc., 43, 92.

Harper A. E., Benevenga N. J., Wohlhueter R. M., 1970. Physiol. Rev., 50, 230-240.

Harper A. E., Miller R. H., Block K. P., 1984. Ann. Rev. Nutr., 4, 409-454.

Lindsay D. B., Buttery P. J., 1980. 125-146. In Buttery P. J. and Lindsay D. B., Protein deposition in animals, Butterworths, London. 